\title{
Phonon assisted tunneling in Josephson junctions
}

\author{
E.G. Maksimov, P.I. Arseyev and N.S. Maslova \\ P.N. Lebedev Physical Institute RAS, Moscow 11 7924, Russia
}

(August 11, 2018)

\begin{abstract}
The expression for additional subgap current in the presence of electronphonon interaction is derived. We show that the phonon assisted tunneling leads to the appearance of peaks on current-voltage characteristics at the Josephson frequencies corresponding to the Raman-active phonons. The relation of the obtained results to the experimental observation are discussed.

72.20 Fg., $74.50 .+\mathrm{r}$
\end{abstract}

Typeset using REVTEX 
It is well known that the superconducting Josephson current at nonzero voltage $V$ across the junction oscillates at a so called Josephson frequency $\omega$ [四]:

$$
\hbar \omega=2 e V
$$

were $V$ is the voltage on the contact.

This alternating $(\mathrm{AC})$ supercurrent can generate time dependent longitudinal or transverse electromagnetic fields within the junction. Nonlinear character of this phenomenon leads to the appearance of many peculiarities in the current-voltage characteristic (CVC) of a Josephson contact [2]. As an example, the CVC can exhibit current peaks, or multiplevalued $\mathrm{CVC}$ with different branches can appear at some voltages. These effects result from the coupling of the Josephson oscillation to electromagnetic cavity modes of the junction structure [3, 4]. Voltages are connected with the corresponding mode frequencies $\omega_{i}$ by the Josephson relation Eq. (11). The possibility of the generation of phonons in Josephson junctions have been predicted by Ivanchenko and Medvedev [5]. The authors have considered the excitation of the long wavelength acoustic resonance modes in the dielectric layer of the contact, which can influence the shape of the CVC of the junction in the same way as excitation of electromagnetic cavity modes. To our knowledge, the resonant current peaks in the CVC and the branching of the CVC, corresponding to the acoustic resonances predicted in [5], have never been observed experimentally. The mere fact of the phonon generation in classical Josephson junctions, caused by the quasiparticle current or by the AC Josephson current, was repeatedly found experimentally [6] [ This generation was observed by measuring phonon emission using phonon spectrometer. Two mechanisms of this generation were discussed: the direct conversion of electromagnetic waves into phonons and phononassisted tunneling processes [8]. Which of the mechanisms better fits experimental situation have not been established at full length.

Recently specific subgap structures in the form of current peaks (resonances) have been observed [9, 10] in the CVC's of intrinsic Josephson junctions in $\mathrm{Bi}_{2} \mathrm{Sr}_{2} \mathrm{Ca}_{1} \mathrm{Cu}_{2} \mathrm{O}_{8}$ and $\mathrm{Tl}_{2} \mathrm{Ba}_{2} \mathrm{Ca}_{2} \mathrm{Cu}_{3} \mathrm{O}_{10}$ with the tunneling current in the $\vec{c}$-direction, perpendicular to the copperoxygen layers. The obtained results have been explained [11,12] by the coupling of the optical phonons in the dielectric layers being the ionic crystal with the AC Josephson current (or, more precisely, with the longitudinal electric field existing inside the dielectric layer). The displacement current contribution $(\dot{D})$ to the total current was described in the framework of a simple macroscopic dielectric function for ionic crystals $\varepsilon(\omega)$. It was shown that peaks in the CVC appear when the voltage satisfies the condition

$$
2 e V=\hbar \omega_{\mathrm{LO}}
$$

where $\omega_{\mathrm{LO}}$ is the frequency of the longitudinal optical (LO) phonons with the wavevector $q=0$. These results can be easily obtained from the equations of Refs. [3, 田 for the electromagnetic waves generation if one substitutes the displacement current $\dot{D}$ in the form:

$$
\dot{D}(t)=i \int \mathrm{d} \omega \omega \varepsilon(\omega) E(\omega) \mathrm{e}^{i \omega t}
$$

The peak appearance corresponds to zero value of the dielectric function, because $\varepsilon\left(\omega_{L O}\right)=$ 0 . 
A fine structure in the CVC of a single $\mathrm{Bi}_{2} \mathrm{Sr}_{2} \mathrm{Ca}_{1} \mathrm{Cu}_{2} \mathrm{O}_{8}$ break junction have been reported earlier [13,14 which could have some resemblance with the subgap structure in BSCCO stacks [9 [1]. The results of the detailed investigations of this structure are presented in the preceding article [15]. The essential result of this work is the observation of the local branching of the CVC and the dips in the $\mathrm{d} I / \mathrm{d} V$ characteristics at the voltages $V$ satisfying the condition

$$
2 e V=\hbar \omega_{\mathrm{R}}
$$

where $\hbar \omega_{\mathrm{R}}$ are the energies of the Raman-active phonons.

The explanation for the observed subgap structure given in Refs. [11.12] is based on direct conversion of the $\mathrm{AC}$ longitudinal electric field, existing inside the junction into LO phonons carrying electric field also inside the junction. But the direct conversion of longitudinal electric field into Raman-active phonons is forbidden due to selection rules. Moreover, the very existence of the well defined dielectric layers in the breakjunctions studied in Ref. [15] is very improbable. Thus "electromagnetic" explanation can not be applied to the case of Raman active phonons, but such phonons can be emitted due to the electron-phonon interaction in the banks of the junction and can interfere with AC Josephson current.

Here we apply the ideas developed in the work [5] to explain the results obtained in Refs. [13 [15]. For this purpose we generalize the approach used in [5] to include interaction of electrons with all phonons and not only with acoustical ones. This generalization is very important for the high-Tc materials, because the range of Josephson frequencies exceeds the range of the main part of the phonon spectra. The total Hamiltonian can be written for this case as

$$
\mathcal{H}_{\text {total }}=\mathcal{H}_{1}+\mathcal{H}_{2}+\left(\sum_{k, p, \sigma} T_{k p} a_{k \sigma}^{+} b_{p \sigma}+\text { h.c. }\right)+\left(\sum_{\substack{k, p, \sigma \\ q, \lambda}} T_{k p} a_{k \sigma}^{+} b_{p \sigma} \alpha_{q \lambda}\left(c_{q \lambda}+c_{-q \lambda}^{+}\right) .+ \text {h.c. }\right)
$$

Here the terms $\mathcal{H}_{1}$ and $\mathcal{H}_{2}$ describe electrons on the two sides of the tunneling junction with the operators $a_{k \sigma}$ and $b_{p \sigma}$ correspondingly. These Hamiltonians include an interelectron interaction leading to superconductivity. We do not consider here this interaction explicitly. It can be the electron-phonon interaction or any other. The only important assumption made is that this interaction leads to the appearance of superconductivity and the Josephson current exists between the two superconductors. We will use the BCS formulas for Josephson current for the case of isotropic order parameter in the main part of the paper. Qualitatively, results are not very sensitive to the symmetry of pairing. It is supposed also that these Hamiltonians include the interaction of the electrons with the bias voltage

$$
-e V_{1}(t) \sum_{k, \sigma} a_{k \sigma}^{+} a_{k \sigma} \quad \text { and } \quad-e V_{2}(t) \sum_{p, \sigma} b_{p \sigma}^{+} b_{p \sigma}
$$

where

$$
V_{1}(t)-V_{2}(t)=V(t)
$$

and $V(t)$ is the bias voltage, applied to the junction. Since we are interested in direct current changes, we can use the usual approximation simplifying the problem. Namely, we 
neglect electrodynamic selfinteraction in the junction, which is quite reasonable for weak AC component of the current (Josephson current) [2]. Then the bias voltage is equal to a given constant external bias $V(t)=V$. The third term in Eq. (5) is the usual tunneling Hamiltonian and $T_{k p}$ is a matrix element for the tunneling of an electron. The fourth term in Eq. (5) describes the phonon assisted tunneling of electrons. The value $\alpha_{q \lambda}$ stands for the electron-phonon coupling and the operators $c_{q \lambda}$ and $c_{-q \lambda}^{+}$describe phonons with the given momentum $q$ and the polarization $\lambda$. A similar expression for this term was used earlier by Kleinman [16] to calculate the contribution of the phonon assisted tunneling to the quasiparticle current between superconductors. We would like to emphasize here that the existence of such a term in the total Hamiltonian does not depend on the origin of the superconductivity in HTSC systems. There is some experimental evidence [17] that the HTSC material possesses considerably strong electron-phonon interaction even though it does not govern totally the superconducting properties of HTSC systems.

Introducing the expression for the total current as

$$
I=e\left\langle\dot{N}_{1}\right\rangle
$$

where $N_{1}$ is the number of electrons in one side of the junction and $e$ is the electron charge

$$
N_{1}=\sum_{k, \sigma} a_{k \sigma}^{+} a_{k \sigma} .
$$

We can write for the current the following expression $(\hbar=1)$

$$
I=-\mathrm{i} e\left\langle\left[N_{1}, \mathcal{H}\right]\right\rangle .
$$

The symbol $\langle\cdots\rangle$ means the averaging over the nonequilibrium statistical ensemble. The last two terms in Eq. (5) give the nonzero contribution to this commutator. Substituting these terms into Eq. (10) we get

$$
I=2 e \operatorname{Im}\left\{\sum_{k, p, \sigma} T_{p k}\left\langle a_{k \sigma}^{+}(t) b_{p \sigma}(t)\right\rangle+\sum_{\substack{k, p, \sigma \\ q, \lambda}} T_{p k} \alpha_{q \lambda}\left\langle a_{k \sigma}^{+}(t) b_{p \sigma}(t)\left(c_{q \lambda}(t)+c_{-q \lambda}^{+}(t)\right)\right\rangle\right\} .
$$

After long but simple calculations following close to that done in the work [5] we get

$$
I(t)=\left(1+\sum_{q, \lambda} \alpha_{q \lambda}\left\langle c_{q \lambda}(t)+c_{-q \lambda}^{+}(t)\right\rangle\right)\left[I_{S}(t)+I_{N}\right]
$$

where $I_{S}(t)$ describes the contribution of the superconducting Josephson current (AC part) to the total current and $I_{N}$ (DC part) gives the contribution of the quasiparticle current. The Josephson current can be written in the form

$$
I_{S}(t)=\operatorname{Re}\left\{I_{p}\left(\frac{\omega_{j}}{2}\right)\right\} \sin \phi(t)+\operatorname{Im}\left\{I_{p}\left(\frac{\omega_{j}}{2}\right)\right\} \cos \phi(t)
$$

where

$$
\omega_{j}=\frac{2 e V}{\hbar} .
$$


The value $\phi(t)$ is the order parameter phase difference between the two sides of the junction:

$$
\phi(t)=\omega_{j} t
$$

We shall not reproduce here the expressions for the values $I_{p}\left(\frac{\omega_{j}}{2}\right)$ and $I_{N}$ which can be found in the works [2, 4, 18]. It is only important for us that $\operatorname{Im}\left\{I_{p}\left(\omega_{j}\right)\right\}$ and $I_{N}(V)$ are very small at low temperature $T$ and at small voltage $V$. For the BCS model $\operatorname{Re}\left\{I_{p}(\omega)\right\}$ at small voltages has the form

$$
\operatorname{Re}\left\{I_{p}\left(\frac{\omega_{j}}{2}\right)\right\} \approx I(0) \approx \frac{\pi}{2} \frac{\Delta(T)}{e R_{\mathrm{N}}} \tanh \frac{\Delta(T)}{2 T}
$$

Here $\Delta$ is the superconducting gap, $e$ is the electron charge and $R_{\mathrm{N}}$ is the resistivity of the contact in the normal state. As $V$ increases the current amplitude $\operatorname{Re}\left\{I_{p}(\omega)\right\}$ increases also and at $V=2 \frac{\hbar}{e} \Delta$ it has the Riedel logarithmic singularity [19] (qualitatively originated from the density of states singularity at the gap edges).

To calculate the total current in the presence of the phonon assisted tunneling we should calculate the value $\left\langle c_{q \lambda}+c_{-q \lambda}^{+}\right\rangle$. Using the Hamiltonian (5) and adding the free phonon part $\mathcal{H}_{\mathrm{ph}}$

$$
\mathcal{H}_{\mathrm{ph}}=\sum_{q, \lambda} \omega_{q \lambda} c_{q \lambda}^{+} c_{q \lambda}
$$

we can obtain the equation of motion in Heisenberg representation for the phonon operator $\left(c_{q \lambda}(t)+c_{-q \lambda}^{+}(t)\right)$. For the averaged values this gives:

$$
\mathcal{D}_{q \lambda}^{-1}(t)\left\langle c_{q \lambda}(t)+c_{-q \lambda}^{+}(t)\right\rangle=-2 \operatorname{Re} \sum_{k, p, \sigma} \int \mathrm{d} r \mathrm{e}^{-\mathrm{i} q r} \alpha_{q \lambda} T_{k p}\left\langle\left(a_{k \sigma}^{+}(t) b_{p \sigma}(t)\right\rangle .\right.
$$

Here $\mathcal{D}_{q \lambda}^{-1}(t)$ is operator inverse to the phonon Green function

$$
\mathcal{D}_{q \lambda}^{-1}(t)=-\frac{1}{\omega_{q \lambda}}\left[\frac{\partial^{2}}{\partial t^{2}}+\omega_{q \lambda}^{2}+\gamma \frac{\partial}{\partial t}\right]
$$

where $\omega_{q \lambda}$ are the phonon frequencies and $\gamma$ is the small phonon relaxation rate. The righthand side of Eq. (18) appears due to phonon-assisted tunneling processes and plays the role of an external source for lattice vibrations. (For the equilibrium case $\left\langle c_{q \lambda}(t)+c_{-q \lambda}^{+}(t)\right\rangle=0$ ). Following again the work [5] we can calculate the contribution to this function from oscillating Josephson current:

$$
\sum_{k, p} \int \mathrm{d} r \mathrm{e}^{\mathrm{i} q r} \alpha_{q \lambda} T_{k p}\left\langle a_{k \sigma}^{+}(t) b_{p \sigma}(t)\right\rangle=\frac{\alpha_{0 \lambda}}{e}\left(I_{p}\left(\frac{\omega_{j}}{2}\right) \mathrm{e}^{\mathrm{i} \phi(t)}\right) \delta_{q, 0}
$$

Since we have neglected the space dependence of the phase $\phi(t)$ only the $q=0$ component gives a nonzero contribution in Eq. (20).

The solution of Eq. (18) can be easily found now:

$$
\left\langle c_{0 \lambda}(t)+c_{0 \lambda}^{+}(t)\right\rangle=-\frac{2 \alpha_{0 \lambda}}{e} \operatorname{Re}\left\{\mathcal{D}_{0 \lambda}\left(\omega_{j}\right) I_{p}\left(\frac{\omega_{j}}{2}\right) \mathrm{e}^{\mathrm{i} \omega_{j} t}\right\}
$$


with phonon Green function $\mathcal{D}_{0 \lambda}(\omega)$ :

$$
\mathcal{D}_{0 \lambda}(\omega)=\frac{\omega_{0 \lambda}}{\omega^{2}-\omega_{0 \lambda}^{2}-\mathrm{i} \omega \gamma}
$$

Now we consider for simplicity the case of low temperature $T$, so only the terms with Re $\left\{I_{p}\right\}$ should be taken into account. In this case Eq. (21) can be written as

$$
\left\langle c_{0 \lambda}(t)+c_{0 \lambda}^{+}(t)\right\rangle=2 \frac{\alpha_{0 \lambda} \omega_{0 \lambda}}{e} \operatorname{Re} I_{p}\left(\frac{\omega_{j}}{2}\right)\left[\operatorname{Im}\left\{\mathcal{D}_{0 \lambda}\left(\omega_{j}\right)\right\} \sin \omega_{j} t-\operatorname{Re}\left\{\mathcal{D}_{0 \lambda}\left(\omega_{j}\right)\right\} \cos \omega_{j} t\right]
$$

Substituting Eq. (23) into Eq. (12) and averaging the oscillating terms over the time, we get the expression for the excess current $\Delta I(V)$. Using the explicit form of $\mathcal{D}_{0 \lambda}\left(\omega_{j}\right)$ (Eq. (22)) we finally obtain :

$$
\Delta I(V)=\sum_{\lambda} \frac{\alpha_{0 \lambda}^{2} \omega_{0 \lambda}}{e} \frac{\gamma \omega_{j}\left\{\operatorname{Re} I_{p}\left(\omega_{j} / 2\right)\right\}^{2}}{\left(\omega_{j}^{2}-\omega_{0 \lambda}^{2}\right)^{2}+\gamma^{2} \omega_{j}^{2}}
$$

where $\omega_{j}$ is defined by Eq. (14). This expression shows that there are peaks in the CVC at the voltages corresponding to the condition

$$
2 e V=\hbar \omega_{0 \lambda}
$$

where $\omega_{0 \lambda}$ are the frequencies of the optical phonons with $q=0$. The value $\mathrm{d} I(V) / \mathrm{d} V$ has correspondingly the dips very close to voltages satisfying Eq. (25). The difference between the peaks positions and the dips is of the order of a small value $\gamma$. Note, that contrary to Eq. (1), now Eq. (25) includes all optical phonons (not only LO) and these are the phonons in superconductors themselves and not in the intermediate dielectric layer. The value of the electron-phonon coupling $\alpha_{0 \lambda}^{2}$ determines how great the contribution from each of the phonon branches is. This value is equal to zero for some phonon modes with $q=0$ due to the symmetry reason. This is the case, for example, for infrared-active modes. As it is well known [20] the coupling constant of electrons with the Raman-active phonons is nonequal to zero even for $q=0$. Moreover, as it was shown by the first principle calculations [21] the constant of coupling with Raman-active phonons is considerably large. This is why the peaks mainly connected with such phonons are observed in the experiments made on break junctions [15].

The current excess can be find for all temperatures $T$ and voltages $V$ numerically using Eq. (12) and Eq. (21) obtained in this work. In the present work we would like only to mention some clear qualitative consequences of such calculations. The peak amplitudes or the dips in the $\mathrm{d} I(V) / \mathrm{d} V$ characteristics - will demonstrate the Riedel peculiarities: the amplitude will increase for the values of $e V / \hbar$ close to $2 \Delta$. This subgap structure will disappear for $\mathrm{eV} / \hbar$ larger than $2 \Delta$, because the value $I_{p}(V)$ quickly goes to zero for $e V / \hbar>2 \Delta$. The amplitude of the peaks decreases with decreasing of the critical Josephson current as $I_{c}^{2}(0)$. We would like to emphasize here that the amplitude of the peaks predicted in the work [11.12] is also proportional to $I_{c}^{2}$ in contradiction with the statement announced in these works. These amplitudes will also change in the external magnetic field due to the change of the value $I_{c}$. 
In conclusion, simple microscopic theory describing the phonon assisted Josephson tunneling was derived. It was shown that there exists subgap excess current. The current excess demonstrates peaks at voltages $V$ such as $2 \mathrm{eV} / \hbar$ is equal to the frequencies of the Ramanactive phonon modes. The amplitudes of the peaks are proportional to $I_{c}^{2}$, the square of the Josephson critical current. All these results are in good agreement with the experimental work [15].

The case of anisotropic pairing (e.g. $d$-pairing) will not change essentially the obtained results, till the AC Josephson current exists. The functions $I_{p}(\omega)$ and $I_{S}(\omega)$ will be certainly different from that of given in the Ref. [2, 4, 18], but all other equations will not be changed. The only necessary things are the existence of the AC Josephson current and the electron-phonon interaction. Therefore the position of the subgap structures on the CVC is determined in any case by the frequencies of Raman-active modes. Of course, the form

of this structure and its temperature dependence can differ from those for isotropic pairing. The problem of numerical calculations of CVC for $T \neq 0$ in different pairing models is now under investigation.

\section{ACKNOWLEDGMENTS}

This work was supported partially by RFBR grants 96-02-16134 , 96-15-96476 and grant 96-081 of the Programm "Superconductivity". One from the authors (E. G. M.) would like to thank the University of Wuppertal and Prof. H. Piel for kind hospitality. We are grateful to Prof. Ya. Ponomarev for the communicating the results [15] to us before publication and for the continuous exchange of information. 


\section{REFERENCES}

[1] B.D. Josephson, Phys. Lett. 1, 251 (1962); Advances in Physics 14, 419 (1966)

[2] K.K. Likharev, Dynamics of Josephson Junctions and Circuits (Gordon and Breach, New York, 1996)

[3] I.O. Kulik, JETP Lett. 2, 84 (1965)

[4] N.R. Werthammer, Phys. Rev. 147, 255 (1966)

[5] Yu.M. Ivanchenko, Yu. V. Medvedev, Sov. Phys.-JETP 33, 1223 (1971)

[6] W. Eisenmenger, A.H. Dayem, Phys. Rev. Lett. 18, 125 (1967)

[7] H. Kinder, Phys. Rev. Lett. 28, 1564 (1972)

[8] P. Berberich, R. Buemann, H. Kinder, Phys Rev. Lett. 49, 1500 (1982)

[9] K. Schlenga, G. Hechtfischer, R. Kleiner, W. Walkenhorst, P. Müller, H.L. Johnson, M. Veith, W. Brodkorb, E. Steinbeiß, Phys. Rev. Lett. 76, 4943 (1996)

[10] R. Kleiner, Journ. Low Temp. Phys. 106, 453 (1997)

[11] Ch. Helm, Ch. Preis, F. Forsthofer, J. Keller, K. Schlenga, R. Kleiner, P. Müller, Phys. Rev. Lett. 79, 737 (1997)

[12] K. Schlenga, R. Kleiner, G. Hechtfischer, M. Mößle, S. Schmitt, P. Müller, Ch. Helm, Ch. Preis, F. Forsthofer, J. Keller, H.L. Johnson, M. Veith and E. Steinbeiß, Phys. Rev. B 57, 14518 (1998)

[13] B.A. Aminov, A.A. Bush, L.I. Leonyuk, T.E. Os'kina, M.V. Pedyash, D.K. Petrov, Ya.G. Ponomarev, H.T. Rakhimov, K. Sethupathi, M.V. Sudakova, Springer Proceedings in Physics, Superconducting Devieces and Their Applications, ed. by H. Koch and H. Lübbig,Vol. 64, pp. 45-48 (1992)

[14] Ya.G. Ponomarev, A.I. Kuzmich, E.B. Tsokur, M.V. Sudakova, S.N. Tchesnokov, B.A. Aminov, M.A. Hein, M.A. Lorenz, G. Müller, H. Piel, V.Z. Kresin, T.E. Os'kina and Yu.D. Tretyakov, (Proceedings of the 5th int. NATO Advanced Research Workshop, Moscow, Russia, March 24-29, 1998)

[15] Ya.G. Ponomarev, E.B. Tsokur, M.V. Sudakova, S.N. Tchesnokov, B.A. Aminov, M.A. Lorenz, G. Müller and H. Piel, (submitted to Phys. Rev. Lett.)

[16] L. Kleinman, Phys. Rev. 132, 2484 (1963)

[17] C. Thomson, Light scattering in Solids 6, 285 (Edid. M. Cardona and G. Güntherodt, Springer Verlag, Heidelberg, 1995)

[18] R.E. Harris, Phys. Rev. B 10, 84 (1974)

[19] E.R. Riedel, Z.Naturforsch. 19a, 1634 (1964)

[20] Yu.E. Kitaev, M.F. Limonov, A.G. Panfilov, R.A. Evarestov and A.P. Mirgorodsky, Phys. Rev. B 49, 9933 (1994)

[21] A.I. Lichtenstein, I.I. Mazin, O.R. Andresen, Phys. Rev. Lett. 74, 2302 (1995) 